\title{
Research on the Method of Frequency Measurement Based on Pulse Width Measurement
}

\author{
Jian Huang \\ XiJing University, Xi'an 710123, China; \\ 565200245@qq.com
}

Keywords: Frequency measurement; Positive pulse width; Duty cycle

\begin{abstract}
In engineering design and experiment, the frequency of square wave is often measured. At present, the commonly used method is the "time sluice" method, which is to measure the frequency of square wave by measuring the jump times of the rising or descending edge of the square wave in 1s. But if the frequency of the square wave is high enough, the processor is required to be fast enough. The high frequency signal is prone to error, so the precision is not high. In order to improve the accuracy, we can use the "pulse width" method to measure the frequency, that is to measure the width of the positive pulse width, and then calculate the frequency according to duty cycle. This method has high accuracy and short measurement time and high efficiency.
\end{abstract}

\section{Introduction}

In engineering practice, it is often necessary to measure the frequency of different waveforms. At present, there are many methods of frequency measurement, in which the pulse width measurement is a common method. The width of the positive pulse width is measured mainly by the timer, then the cycle is calculated according to the duty ratio, and the frequency is determined according to the cycle. In this method, the faster the processor is, the higher the frequency of measurement and the higher the accuracy. Taking STM32F103ZET6 as an example, the main frequency is $72 \mathrm{MHz}$, and the highest frequency can be up to $900 \mathrm{KHz}$.

\section{Hardware Design}

The pulse width of the STM32F103ZET6 is captured as shown in Figure 1. Figure 1 will be the introduction of external signal connected to the timer pin, when the signal changes from negative to positive pulse pulse jump, start the timer started until the stop time is negative pulse pulse to jump, and this time is the time according to the pulse width and duty cycle you can get the measured waveform and the countdown, you can get the frequency.

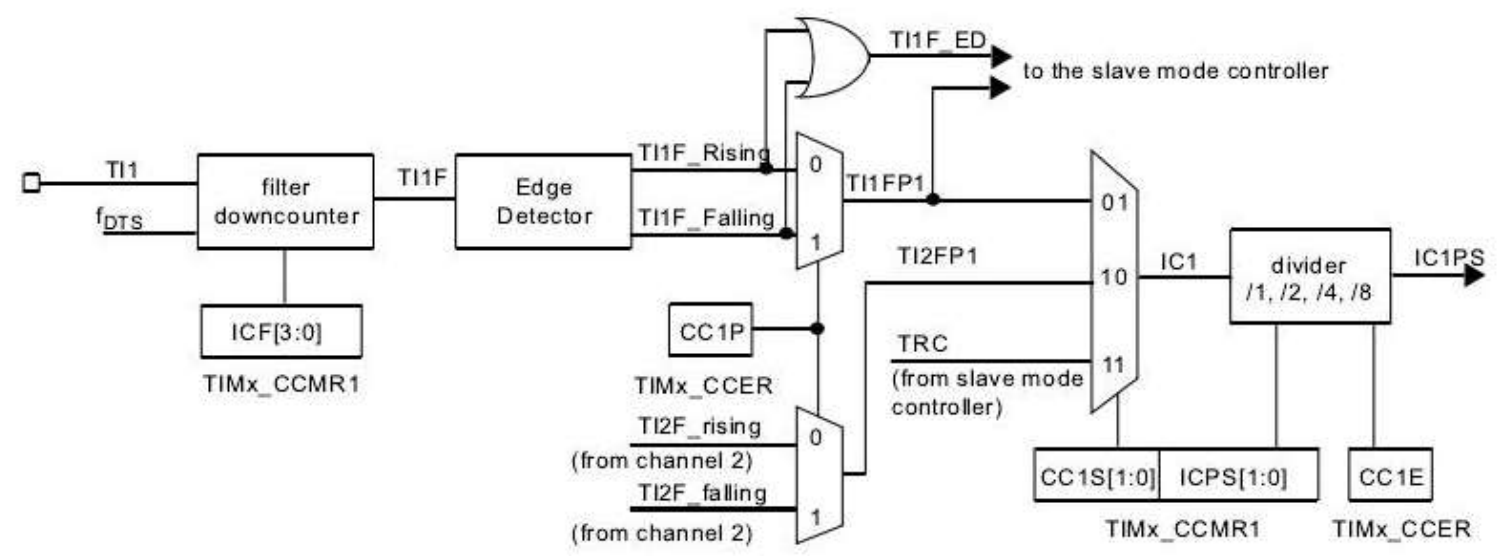

Figure 1 Capture/compare channel 


\section{Software Program}

In this design, it is programmed in the C language under KEIL 5. Realize the measurement of the pulse width is using the timer, because the duty ratio is $50 \%$, so it is multiplied by 2 , then the countdown, you can get the measured signal frequency.

The read frequency code is as follows:

if(TIM2CH1_CAPTURE_STA\&0X80)

$$
\{
$$

temp=TIM2CH1_CAPTURE_STA\&0X3F;

temp* $=65536$;

temp+=TIM2CH1_CAPTURE_VAL;

printf("HIGH:\%d us\rın",temp/72);

TIM2CH1_CAPTURE_STA=0;

\}

The code for measuring the frequency is as follows:

void TIM2_IRQHandler(void)

\{

u16 tsr;

tsr=TIM2->SR;

if((TIM2CH1_CAPTURE_STA\&0X80)==0)

\{

if(tsr\&0X01)

\{

if(TIM2CH1_CAPTURE_STA\&0X40)

\{

if $\left(\left(T I M 2 C H 1 \_C A P T U R E \_S T A \& 0 X 3 F\right)==0 X 3 F\right)$

\{

TIM2CH1_CAPTURE_STA|=0X80;

TIM2CH1_CAPTURE_VAL=0XFFFF;

\}

else TIM2CH1_CAPTURE_STA++;

\}

if $(\operatorname{tsr} \& 0 x 10)$

\{

if(TIM2CH1_CAPTURE_STA\&0X40)

\{

TIM2CH1_CAPTURE_STA| $=0 X 80$;

TIM2CH1_CAPTURE_VAL=TIM2->CCR4;

TIM2->CCER\& $=\sim(1<<13)$;

\}else

\{

TIM2CH1_CAPTURE_VAL $=0$;

TIM2CH1_CAPTURE_STA=0X40;

TIM2->CNT $=0$;

TIM2->CCER $\mid=1<<13$;

\}\}\}

TIM2->SR=0;

\} 


\section{Summary}

This paper describes the method of using the pulse width method to measure the frequency, and gives the hardware circuit and the specific program. According to the test results, the measurement accuracy can reach 1US, which is suitable for the low and low frequency signals in the measurement. If the frequency range of the measurement is to be increased, the main frequency of the processor must be increased. This method has certain practical value, and has high precision, short time and high efficiency.

\section{Reference}

[1] Lv Yunfang, Chen Shuaishuai, Hao Xingsen, Zhang Hao. The design and implementation of [J] intelligent tracking car C51 advanced language program based on the control of laboratory research and exploration,.2015,34 (3): 142-145.

[2] Chen life method, Wang Zhongpeng. Improved design of synchronous gate circuit, such as precision frequency measurement, [J]. electrical measurement and instrument, 2015,52 (18): 90-95

[3] Zhang Tianheng, Ye Wei and so on. A portable high precision frequency meter based on SOPC design [J]. instrument technology and sensor, 2015,5:34-37.

[4] Jiangxiu, sub branch, Zhang Huanchun. Using equal precision frequency measurement method to realize vibration string sensor frequency measurement [J]. sensor technology, 2001,20 (6): 53-55

[5] Huang Guogang, Chen Ming, Zhang Peng. High precision frequency measurement of surface acoustic wave CO gas sensor [J]. computer measurement and control, 2009:17 (6):1033-1038

[6] Shen Wei, Wang Junzheng, Wang Zheng Jun. A method of frequency measurement based on signal compensation, [J]. instrument and instrument, 2010,31 (10): 2192-2197.

[7] Liu Ya, Li Xiaohui, Wang Yulan. A multi-channel frequency measurement system based on digital technology [J]. instrument and instrument, 2009,30 (9):1963-1968

[8] Peng L, Shizhao N, Zheng W, Ziwei J, Jianwu Y, Zhongxiang Q, Wangmo P. Predicting durations of online collective actions based on Peaks' heights [J]. Communications in Nonlinear Science and Numerical Simulation. 2018, 55: 338-354.

[9] Wang Zhengjun, Pan Lei, Ji Rui. Frequency measurement method of discrete phase difference detection based on [J]. Chinese Journal of scientific instrument, 2013,34 (5): 994-999. 